\title{
Ethical examination of sham surgeries for relief of chronic pain in clinical practice
}

\author{
Katherine Fleshner, Jacek Orzylowski
}

\begin{abstract}
Treatment of chronic pain is challenging for both patients and physicians alike. Interventional management of pain is often indicated for patients who are not helped by pharmacotherapy, and can include procedures such as neurectomy and vertebroplasty. However, randomized controlled trials of these procedures often demonstrate a significant improvement in symptomology among patients in the control arm who have instead undergone a sham surgery, which eliminates the perceived surgical steps required for benefit but mimics the surgery in every other way. We examine whether an ethical framework might exist for sham surgeries to hypothetically be performed for clinical benefit of chronic pain. Once all evidence-based options are exhausted, performing sham surgeries may be justified under beneficence and non-maleficence since sham procedures are often equally efficacious but considerably safer than their true intervention counterparts. Physicians must only recommend such procedures with the intent of ameliorating patient suffering. Some degree of disclosure of a possible placebo effect prior to a sham surgery may satisfy the principle of autonomy while still maintaining the placebo response.
\end{abstract}

\section{INTRODUCTION}

Chronic pain is a challenging condition. It is notoriously difficult to treat effectively and carries a large disease burden. ${ }^{1}$ Unlike acute pain, chronic pain may or may not be associated with trauma and may be persistent. Chronic pain may also manifest with no clear etiology despite full clinical and radiological workup. ${ }^{1}$

Current modalities of treatment for chronic pain include physical therapy, pharmacotherapy, and interventional therapy. ${ }^{2}$ Pharmacotherapy is typically the first-line of treatment and includes non-steroidal anti-inflammatory drugs and opioids. ${ }^{2}$ Interventional therapy is a newer modality of therapy often reserved for drugresistant chronic pain. ${ }^{3}$ Interventions include implantable devices such as analgesic infusion pumps, spinal cord stimulation, and surgeries such as neurectomy and vertebroplasty. ${ }^{2}$

Novel surgical techniques are rarely rigorously evaluated before they are performed on patients. ${ }^{4}$ Nevertheless, surgical trials to evaluate the effectiveness of pain procedures will often utilize two patient groups: one group receives the active technique being studied and another receives a sham surgery. ${ }^{5}$ A sham surgery is defined as a surgery that mimics everything about the active procedure except for the steps considered necessary for benefit. ${ }^{5}$

Despite the lack of active intervention, pain procedure trials often report clinically significant improvements in pain in the sham surgery arm. ${ }^{5}$ Sham surgeries have also produced a marked improvement in disability and quality of life. ${ }^{5}$ Furthermore, evaluation of surgical trials with sham controls found improvement in the sham group in $74 \%$ of trials. ${ }^{6}$ For this reason, sham surgeries are an important component of research trials as they help to elucidate whether or not procedures provide any true benefit.

In spite of their importance in the research setting, sham surgeries are not routinely performed on patients in the clinical setting. In fact, surgical procedures found to have no measurable effect beyond placebo are often abandoned altogether due to a preference for evidence-based procedures. ${ }^{7}$ However, in a hypothetical scenario, might there be a way to perform sham procedures for clinical benefit while still maintaining ethical practice? The following article will examine this question under the medical ethical principles of beneficence, non-maleficence and autonomy.

\section{THE PLACEBO EFFECT}

The placebo effect is the symptomatic improvement with the administration of a medication or procedure that, perhaps unbeknownst to the patient, consists of no active ingredient or measure that can produce the anticipated change. ${ }^{8}$ In the era of medical paternalism, physicians regularly prescribed placebos when patients were terminally ill or when indicated therapies were likely to do more harm than good. ${ }^{8,9}$ Advocates of placebo administration also believed that it helped patients psychologically cope with the burden of illness. ${ }^{9}$

There are three dominant theories about how the placebo effect elicits analgesia. One theory is that placebo stimulates endogenous opioids in the body to produce pain relief, which is supported by the fact that placebo analgesia is reduced with administration of an opioid antagonist. ${ }^{10}$ Another theory postulates that operant conditioning of being treated by a physician causes the placebo effect. ${ }^{10}$ This theory can also explain the phenomenon of increased placebo response with increased surgical invasiveness. ${ }^{11}$ Finally, the 'meaning model' of placebo effect suggests that the therapeutic interaction itself and the physician-patient relationship contribute to the placebo response. ${ }^{10}$

\section{BENEFICENCE, NON-MALEFICENCE AND AUTONOMY}

Medical ethics, as delineated by Beauchamp and Childress, consists of four principles: autonomy, beneficence, justice and non-maleficence. ${ }^{12}$ Respect for patient autonomy requires that physicians honor patients' right to self-determination with respect to medical decision-making. ${ }^{12}$ Beneficence states that physicians must always act to promote the health of their patients, while nonmaleficence states that the physician should do no harm to their 
patients. ${ }^{12}$ Finally, the principle of justice is concerned with fairness and equality in the distribution of medical resources. ${ }^{12}$ In this paper, beneficence and non-maleficence will be applied to evaluate whether sham surgeries should be considered in clinical practice. The principle of autonomy will be applied to examine whether physicians should withhold information about the true nature of sham procedures.

The ethical tenet of non-maleficence stems from the Hippocratic Oath, in which the physician vows to do no harm to his or her patients. ${ }^{12}$ Simplistically, it can be argued that the benefits of a therapy must outweigh the risks or adverse effects, and no intervention that brings undue harm to a patient can be justified. With placebo pills, patients risk clinical deterioration but there are no risks associated with the placebo itself. A sham surgery mimics a true surgery, meaning that anesthesia must be administered and a superficial incision made, which are not risk-free. ${ }^{13}$ However, these risks might be offset by the fact that surgery-associated placebo is more potent than drug-associated placebo and thus can provide more benefit. ${ }^{14}$ Sham surgeries are also safer than their true intervention counterparts in RCTs, with far fewer adverse events reported from clinical trials. ${ }^{6}$ Finally, since there is no active intervention, local anesthesia or light sedation and minimally invasive surgical techniques could be employed to reduce the risks associated with anesthesia and the surgical wound. ${ }^{13}$

Similar to non-maleficence, beneficence espouses that physicians should always promote health and act in the best interests of their patients. In this context, beneficence can be interpreted as the duty of the physician to prescribe therapies with demonstrable benefit. Treating chronic pain, however, is rarely so straightforward. Some pain does not respond to pharmacotherapy, and there is wide variation in analgesic response among patients. ${ }^{15,16}$ Also, many of the widely adopted procedures, when tested in RCTs, demonstrate minimal or no added benefit beyond that of a sham surgery.The evidence for various pain surgeries such as arthroscopy and vertebroplasty is, at best, mixed. ${ }^{11,17-19}$ If some pain procedures are no better than placebo, perhaps there can be a hypothetical role for a safer, less invasive placebo to be used.

However, even if sham surgeries are equally efficacious, can the ethical physician offer a placebo without violating beneficence? Is it still an act of deception if the physician does not lie about the nature of the surgery but withholds the fact that it's placebo? Gold and Lichtenberg argue that the mindset of the physician must be considered. ${ }^{20}$ If a physician prescribes a placebo surgery because he or she does not believe in the validity of the patient complaint and/or just seeks to relieve him or herself of a demanding patient - this physician is not acting in the best interest of the patient and beneficence is thus violated. ${ }^{20}$ On the other hand, if the patient has exhausted other options and the physician truly, non-selfishly believes the sham surgery might provide some relief, the physician can still be in accordance with the beneficence principle. ${ }^{20}$

Autonomy suggests that patients, as moral beings, have the right to self-determination in medical decision-making. ${ }^{12}$ Whether prescribing sham therapies can be done while preserving autonomy depends, to some extent, on the degree of disclosure considered necessary to fully respect patient autonomy. This can be difficult to navigate, as studies evaluating patient attitudes regarding physician recommendation of placebo have demonstrated that some patients deem it untrustworthy practice, while others feel it is acceptable..$^{21-23}$ It is generally established, however, that the physician should disclose any information that a reasonable person would want to know and that might influence decision-making; however, there is debate over what exactly this constitutes. In the case of sham surgeries, the physician must communicate the potential risks, but is he or she obligated to explain exactly how the procedure works? To this point, Gold and Lichtenberg argue that patients generally seek care in order to feel better, not to be taught about how things work, rendering this type of information as peripheral. ${ }^{20}$

What if the physician voluntarily discloses the nature of the placebo? If the patient consents to a sham surgery knowing their pain relief may be due to a placebo effect, then the principle of autonomy may be upheld. In fact, there is evidence that disclosure of the possibility of a placebo effect may not completely abolish the response. $^{24,25}$ Physicians must, however, be savvy at communicating the evidence and risks for sham procedures as well as managing patient expectations of improvement so as not to promote false hope or give credence to other unscientific therapies. ${ }^{24,26}$ Bystad et al outline four points that physicians should communicate to patients when discussing placebo procedures. The physician should (1) describe the basic mechanisms of action, (2) provide information demonstrating the possibility of benefit if such information is accurate, (3) maintain a warm, empathic demeanor throughout the encounter, and (4) alleviate the patient's stress as much as possible. ${ }^{27}$ Education about the evidence surrounding sham procedures ensures that patients can make informed decisions about whether or not to proceed.

\section{CONCLUSION}

Chronic pain is difficult to treat. In a hypothetical situation, performing sham surgeries may be justified if the physician's intent is to help the patient, other options have been exhausted and risks are minimized. Some degree of disclosure about the nature of the procedure may be appropriate to uphold patient autonomy and may not abolish the placebo response. The principles of beneficence, non-maleficence and autonomy can provide a hypothetical ethical framework for sham surgeries in clinical practice.

\section{REFERENCES}

1. Grichnik KP, Ferrante FM. The difference between acute and chronic pain. Mt Sinai J Med. 1991;58(3):217-20.

2. Turk DC, Wilson HD, Cahana A. Treatment of chronic non-cancer pain. Lancet. 2011;377(9784):2226-35.

3. Krames ES. Interventional pain management. Appropriate when less invasive therapies fail to provide adequate analgesia. Med Clin North Am. 1999;83(3):787-808, vii-viii.

4. Horng S, Miller FG. Is placebo surgery unethical? N Engl J Med. 2002;347(2):137-9.

5. Gu AP, Gu CN, Ahmed AT, et al. Sham surgical procedures for pain 
intervention result in significant improvements in pain: systematic review and meta-analysis. J Clin Epidemiol. 2017;83:18-23.

6. Wartolowska K, Judge A, Hopewell S, et al. Use of placebo controls in the evaluation of surgery: systematic review. BMJ. 2014;348:g3253.

7. Miller FG. The enduring legacy of sham-controlled trials of internal mammary artery ligation. Prog Cardiovasc Dis. 2012;55(3):246-50.

8. Doherty M, Dieppe P. The "placebo" response in osteoarthritis and its implications for clinical practice. Osteoarthritis Cartilage. 2009;17(10):1255-62.

9. Kaptchuk TJ. Powerful placebo: the dark side of the randomised controlled trial. Lancet. 1998;351(9117):1722-5

10. Hrobjartsson A. The uncontrollable placebo effect. Eur J Clin Pharmacol. 1996;50(5):345-8.

11. Jonas WB, Crawford C, Colloca L, et al. To what extent are surgery and invasive procedures effective beyond a placebo response? A systematic review with meta-analysis of randomised, sham controlled trials. BMJ Open. 2015;5(12):e009655.

12. Gillon R. Medical ethics: four principles plus attention to scope. BMJ. 1994;309(6948):184-8.

13. Wolf BR, Buckwalter JA. Randomized surgical trials and "sham" surgery: relevance to modern orthopaedics and minimally invasive surgery. Iowa Orthop J. 2006;26:107-11.

14. Kaptchuk TJ, Stason WB, Davis RB, et al. Sham device v inert pill: randomised controlled trial of two placebo treatments. BMJ. 2006;332(7538):391-7.

15. McCormick WC, Schreiner RL. Diagnosis and treatment of opiateresistant pain in advanced AIDS. West J Med. 2001;175(6):408-11.

16. Woolf CJ, Mannion RJ. Neuropathic pain: aetiology, symptoms, mechanisms, and management. Lancet. 1999;353(9168):1959-64.

17. Kallmes DF, Comstock BA, Heagerty PJ, et al. A randomized trial of vertebroplasty for osteoporotic spinal fractures. N Engl J Med. 2009;361(6):569-79.

18. Papanastassiou ID, Phillips FM, Van Meirhaeghe J, et al. Comparing effects of kyphoplasty, vertebroplasty, and non-surgical management in a systematic review of randomized and non-randomized controlled studies. Eur Spine J. 2012;21(9):1826-43.

19. Louw A, Diener I, Fernandez-de-Las-Penas C, et al. Sham Surgery in Orthopedics: A Systematic Review of the Literature. Pain Med. 2017;18(4):736-50

20. Gold A, Lichtenberg P. The moral case for the clinical placebo. J Med Ethics. 2014;40(4):219-24

21. Bishop FL, Aizlewood L, Adams AE. When and why placeboprescribing is acceptable and unacceptable: a focus group study of patients' views. PLoS One. 2014;9(7):e101822.

22. Ortiz R, Chandros Hull S, Colloca L. Patient attitudes about the clinical use of placebo: qualitative perspectives from a telephone survey. BMJ Open. 2016;6(4):e011012.

23. Hull SC, Colloca L, Avins A, et al. Patients' attitudes about the use of placebo treatments: telephone survey. BMJ. 2013;347:f3757.

24. Blease C, Colloca L, Kaptchuk TJ. Are open-Label Placebos Ethical? Informed Consent and Ethical Equivocations. Bioethics. 2016;30(6):407-14.

25. Miller FG, Colloca L. The legitimacy of placebo treatments in clinical practice: evidence and ethics. Am J Bioeth. 2009;9(12):39-47.

26. Annoni M, Miller FG. Placebos in clinical practice: an ethical overview. Douleur et Analgésie. 2014;27(4):215-20.

27. Bystad M, Bystad C, Wynn R. How can placebo effects best be applied in clinical practice? A narrative review. Psychol Res Behav Manag. 2015;8:41-5

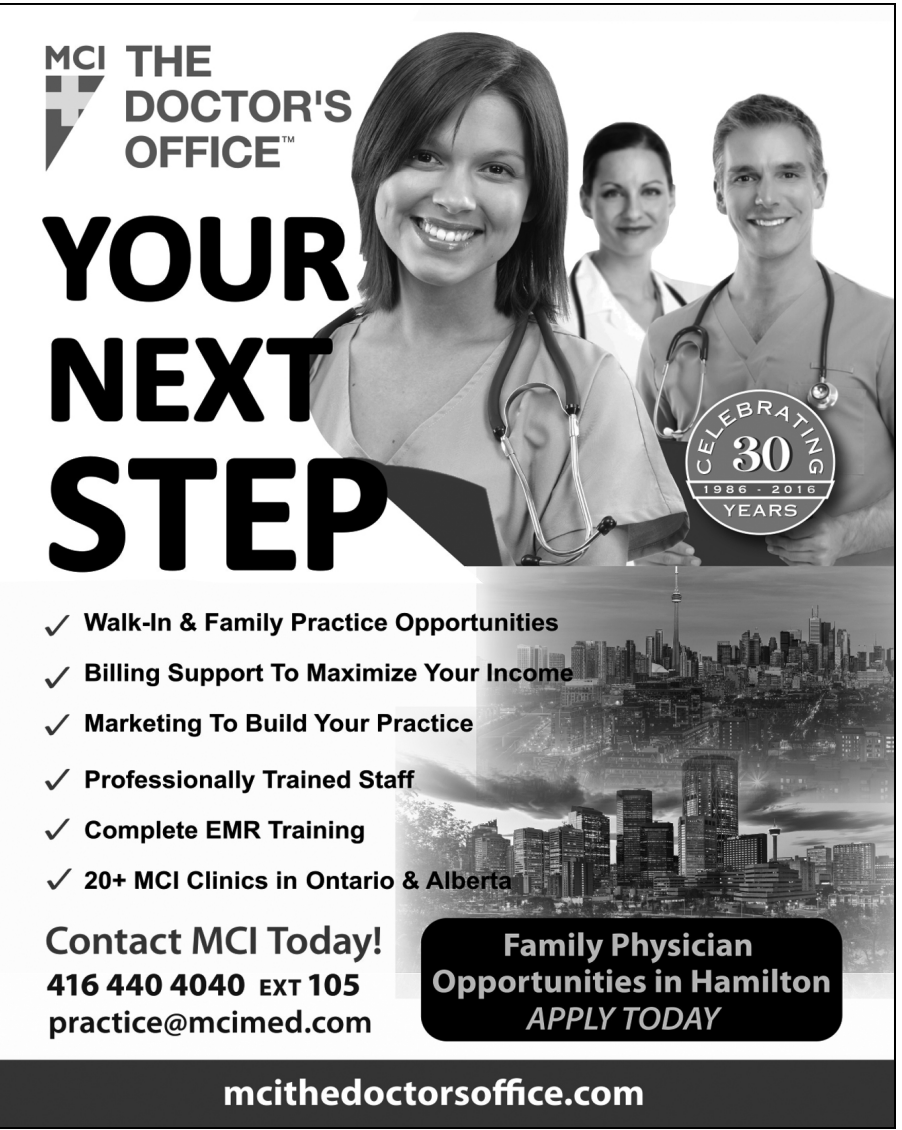

